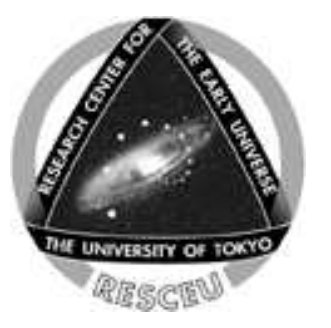

\author{
THE UNIVERSITY OF TOKYO \\ Research Center for the Early Universe RESCEU-33/96 \\ UTAP-240/96
}

\title{
The finite size effect of galaxies on the cosmic virial theorem and the pairwise peculiar velocity dispersions
}

\author{
Yasushi Suto $^{1,2}$ and Yi-Peng Jing ${ }^{1,3}$ \\ ${ }^{1}$ Max-Planck-Institut für Astrophysik, Karl-Schwarzschild-Strasse 1, \\ 85748 Garching, Germany \\ 2 permanent address: Department of Physics and RESCEU \\ (Research Center for the Early Universe), School of Science, \\ The University of Tokyo, Tokyo 113, Japan \\ 3 address after November 1, 1996. RESCEU (Research Center for the Early Universe), \\ School of Science, The University of Tokyo, Tokyo 113, Japan \\ e-mail: suto@phys.s.u-tokyo.ac.jp, jing@utaphp2.phys.s.u-tokyo.ac.jp
}

Received 1996 August 22; accepted __ 


\begin{abstract}
We discuss the effect of the finite size of galaxies on estimating small-scale relative pairwise peculiar velocity dispersions from the cosmic virial theorem (CVT). Specifically we evaluate the effect by incorporating the finite core radius $r_{c}$ in the two-point correlation function of mass, i.e. $\xi_{\rho}(r) \propto\left(r+r_{c}\right)^{-\gamma}$ and the effective gravitational force softening $r_{s}$ on small scales. We analytically obtain the lowest-order correction term for $\gamma<2$ which is in quantitative agreement with the full numerical evaluation. With a nonzero $r_{s}$ and/or $r_{c}$ the cosmic virial theorem is no longer limited to the case of $\gamma<2$. We present accurate fitting formulae for the CVT predicted pairwise velocity dispersion for the case of $\gamma>2$. Compared with the idealistic point-mass approximation $\left(r_{s}=r_{c}=0\right)$, the finite size effect can significantly reduce the small-scale velocity dispersions of galaxies at scales much larger than $r_{s}$ and $r_{c}$. Even without considering the finite size of galaxies, nonzero values for $r_{c}$ are generally expected, for instance, for cold dark matter (CDM) models with a scale-invariant primordial spectrum. For these CDM models, a reasonable force softening $r_{s} \leq 100 h^{-1} \mathrm{kpc}$ would have rather tiny effect. We present the CVT predictions for the small-scale pairwise velocity dispersion in the CDM models normalized by the COBE observation. The implication of our results for confrontation of observations of galaxy pair-wise velocity dispersions and theoretical predictions of the CVT is also discussed.
\end{abstract}

Subject headings: cosmology: theory — large-scale structure of the universe methods: statistical 


\section{INTRODUCTION}

The three-dimensional distribution of galaxies observed in redshift surveys differs from the true one since the distance to each galaxy cannot be determined by its redshift $z$ only; for $z \ll 1$ the peculiar velocity of galaxies, typically $\sim(100-1000) \mathrm{km} / \mathrm{sec}$, contaminates the true recession velocity of the Hubble flow (e.g., Davis \& Peebles 1983; Kaiser 1987; Hamilton 1992), while the true distance for objects at $z \gtrsim 1$ sensitively depends on the (unknown and thus assumed) cosmological parameters. This hampers the effort to understand the true distribution of large-scale structure of the universe. Nevertheless such redshift-space distortion effects are quite useful since through the detailed theoretical modeling, one can derive the peculiar velocity dispersions of galaxies as a function of separation (Davis \& Peebles 1983), and also can infer the cosmological density parameter $\Omega_{0}$ and the dimensionless cosmological constant $\lambda_{0}$, for instance (Ballinger, Peacock \& Heavens 1996; Matsubara \& Suto 1996; Suto \& Matsubara 1996).

Theoretically, a conventional tool to predict small-scale relative pairwise peculiar velocity dispersions is the cosmic virial theorem (Peebles 1976; hereafter CVT). In its simplest form, the prediction is based on the idealistic assumption that galaxies are treated as point particles. As Peebles (1976) remarked, however, the finite size effect is very significant on scale $\sim 1 h^{-1} \mathrm{Mpc}$ even if one considers a radius of $(20 \sim 100) h^{-1} \mathrm{kpc}$ for typical galactic halos. In this paper we explore this realistic effect in more details and discuss its important impact on the application of the CVT to real observations. We will also give reliable predictions for the small-scale velocity dispersions of galaxies in COBE normalized CDM models.

The plan of the paper is as follows; section 2 briefly describes the CVT both in its simplest and in its more realistic form. The finite size effect is considered in $\S 3$, first based on a perturbation theory (§3.1) and then on a full numerical analysis ( 33.2$)$. In $\S 4$ we predict velocity dispersion of galaxies on small scales for COBE normalized CDM models. Discussion and implications of our result, and our main conclusions are given in $\S 5$. 


\section{COSMIC VIRIAL THEOREM}

Assuming that galaxy pairs are gravitationally in "statistical equilibrium" on small scales, Peebles $(1976,1980)$ derived an expression for their relative (one-dimensional) peculiar velocity dispersion as a function of their separation $r$ :

$$
\left\langle v_{12}^{2}(r)\right\rangle_{C V T}=\frac{6 G \bar{\rho}}{\xi_{\rho}(r)} \int_{r}^{\infty} \frac{d r^{\prime}}{r^{\prime}} \int d \mathbf{z} \frac{\mathbf{r}^{\prime} \cdot \mathbf{z}}{z^{3}} \zeta_{\rho}\left(r^{\prime}, z,\left|\mathbf{r}^{\prime}-\mathbf{z}\right|\right),
$$

where $\bar{\rho}$ is the mean density of the universe, and $\xi_{\rho}(r)$ and $\zeta_{\rho}$ are the two- and three-point correlation functions of mass.

The observed two- and three-point correlation functions of galaxies, $\xi_{\mathrm{g}}$ and $\zeta_{\mathrm{g}}$, are well approximated by the following forms (Groth \& Peebles 1977; Davis \& Peebles 1983):

$$
\begin{aligned}
\xi_{\mathrm{g}}(r) & =\left(\frac{r_{0}}{r}\right)^{\gamma}, \\
\zeta_{\mathrm{g}}\left(r_{1}, r_{2}, r_{3}\right) & =Q_{\mathrm{g}}\left[\xi_{\mathrm{g}}\left(r_{1}\right) \xi_{\mathrm{g}}\left(r_{2}\right)+\xi_{\mathrm{g}}\left(r_{2}\right) \xi_{\mathrm{g}}\left(r_{3}\right)+\xi_{\mathrm{g}}\left(r_{3}\right) \xi_{\mathrm{g}}\left(r_{1}\right)\right]
\end{aligned}
$$

where $r_{0}=(5.4 \pm 0.3) h^{-1} \mathrm{Mpc}, \gamma=1.77 \pm 0.04, Q_{\mathrm{g}}=1.29 \pm 0.21(h$ is the dimensionless Hubble constant $H_{0}$ in units of $100 \mathrm{~km} \mathrm{~s}^{-1} \mathrm{Mpc}^{-1}$ ). Thus it is reasonable to assume that the two- and three-point correlation functions of mass, $\xi_{\rho}$ and $\zeta_{\rho}$, also obey the same scaling except for the overall amplitudes:

$$
\xi_{\rho}(r)=\frac{1}{b_{\mathrm{g}}^{2}} \xi_{\mathrm{g}}(r), \quad \zeta_{\rho}\left(r_{1}, r_{2}, r_{3}\right)=\frac{Q_{\rho}}{Q_{\mathrm{g}} b_{\mathrm{g}}^{4}} \zeta_{\mathrm{g}}\left(r_{1}, r_{2}, r_{3}\right) .
$$

Then a straightforward computation yields

$$
\left\langle v_{12}^{2}(r)\right\rangle_{C V T}=6 G \bar{\rho} Q_{\rho} r_{0}^{\gamma} r^{2-\gamma} I_{0}(\gamma) / b_{g}^{2},
$$

where

$$
\begin{aligned}
& I_{0}(\gamma) \equiv \frac{\pi}{(\gamma-1)(2-\gamma)(4-\gamma)} \\
& \times \int_{0}^{\infty} d x \frac{1+x^{-\gamma}}{x^{2}}\left\{(1+x)^{4-\gamma}-|1+x|^{4-\gamma}-(4-\gamma) x\left[(1+x)^{2-\gamma}+|1+x|^{2-\gamma}\right]\right\}
\end{aligned}
$$

Numerically $I_{0}(1.65) \sim 25.4, I_{0}(1.8) \sim 33.2$, and $I_{0}(1.95) \sim 55.6$. More specifically the CVT predicts the small-scale peculiar velocity dispersion as (Peebles 1976; Suto 1993)

$$
\left\langle v_{12}^{2}(r)\right\rangle_{C V T}^{1 / 2}=1460 \sqrt{\frac{\Omega_{0} Q_{\rho}}{1.3 b_{\mathrm{g}}^{2}}} \sqrt{\frac{I_{0}(\gamma)}{33.2}} 5.4^{\frac{\gamma-1.8}{2}}\left(\frac{r_{0}}{5.4 h^{-1} \mathrm{Mpc}}\right)^{\frac{\gamma}{2}}\left(\frac{r}{1 h^{-1} \mathrm{Mpc}}\right)^{\frac{2-\gamma}{2}} \mathrm{~km} / \mathrm{sec} .
$$


The above simple estimate can be improved more realistically by including the finite size of the galaxies, which suppresses the effective gravitational force between pairs, and also possibly changes the behavior of the two-point correlation functions on small scales. In fact Peebles (1976) already remarked that the above two effects may change the predictions of the velocity dispersions significantly. More recently Bartlett \& Blanchard (1996) also discussed the importance of the local mass distribution around pairs in the CVT. Our principal aim here is to describe these effects more extensively using both the perturbation analysis and the full numerical integration. For this purpose we take account of the former effect by softening the gravitational force according to the Plummer law with the softening radius $r_{s}$. Then equation (11) is modified as

$$
\left\langle v_{12}^{2}(r)\right\rangle_{C V T}=\frac{6 G \bar{\rho}}{\xi_{\rho}(r)} \int_{r}^{\infty} \frac{d r^{\prime}}{r^{\prime}} \int d \mathbf{z} \frac{\mathbf{r}^{\prime} \cdot \mathbf{z}}{\left(z^{2}+r_{s}^{2}\right)^{3 / 2}} \zeta_{\rho}\left(r^{\prime}, z,\left|\mathbf{r}^{\prime}-\mathbf{z}\right|\right) .
$$

We model the latter effect by incorporating a nonzero core radius $r_{c}$ as follows:

$$
\xi_{\mathrm{g}}(r)=\left(\frac{r_{0}}{r+r_{c}}\right)^{\gamma}
$$

The two modifications can be still described in equation (7) simply by replacing $I_{0}(\gamma)$ with

$$
\begin{aligned}
& I(y, w ; \gamma) \equiv(1+y)^{\gamma} \int \frac{d \mathbf{t}}{\left(t^{2}+w^{2}\right)^{3 / 2}} \int_{1}^{\infty} \frac{d s}{s} \frac{\mathbf{s} \cdot \mathbf{t}}{(|\mathbf{s}-\mathbf{t}|+y)^{\gamma}}\left[\frac{1}{(s+y)^{\gamma}}+\frac{1}{(t+y)^{\gamma}}\right] \\
& =2 \pi(1+y)^{\gamma} \int_{0}^{\infty} \frac{t^{3} d t}{\left(t^{2}+w^{2}\right)^{3 / 2}} \int_{1}^{\infty} d s\left[\frac{1}{(s+y)^{\gamma}}+\frac{1}{(t+y)^{\gamma}}\right] K(s, t, y ; \gamma),
\end{aligned}
$$

where $y \equiv r_{c} / r, w \equiv r_{s} / r$, and

$$
\begin{aligned}
K(s, t, y ; \gamma) & \equiv \int_{-1}^{1} \frac{\mu d \mu}{\left(\sqrt{s^{2}+t^{2}-2 s t \mu}+y\right)^{\gamma}} \\
& =\frac{(s+t+y)^{4-\gamma}-(|s-t|+y)^{4-\gamma}}{2 s^{2} t^{2}(\gamma-4)} \\
& +\frac{3 y}{2 s^{2} t^{2}(3-\gamma)}\left[(s+t+y)^{3-\gamma}-(|s-t|+y)^{3-\gamma}\right] \\
& +\frac{s^{2}+t^{2}-3 y^{3}}{2 s^{2} t^{2}(2-\gamma)}\left[(s+t+y)^{2-\gamma}-(|s-t|+y)^{2-\gamma}\right] \\
& +\frac{y\left(y^{2}-s^{2}-t^{2}\right)}{2 s^{2} t^{2}(1-\gamma)}\left[(s+t+y)^{1-\gamma}-(|s-t|+y)^{1-\gamma}\right]
\end{aligned}
$$

The integral $I(y, w ; \gamma)$ is divergent for $\gamma>2$ if $y=w=0$. Nonzero values for $y$ and/or $w$ make $I(y, w ; \gamma)$ finite for $\gamma>2$, therefore incorporating the finite size of galaxies in this 
way widens the applicability of the CVT compared with its simplest form (7). For realistic CDM models, the mass two-point correlation functions on small scales may be better described by equation (9) with $\gamma \gtrsim 2$ and $r_{c}>0$, as will be seen below.

\section{EFFECT OF THE SIZE OF GALAXIES}

\section{1. $\quad$ perturbation analysis for $\gamma<2$}

In the case of $\gamma<2$, the integral $I(y, w ; \gamma)$ converges even for $y=w=0$. Then one may treat the effect of nonzero $y$ and $w$ via perturbation theory. Consider the case of $w=0$ first. Let us set $y=u^{\alpha}$ ( $\alpha$ is a parameter greater than unity and will be determined below), and try to find the Taylor expansion with respect to $u$ :

$$
I\left(u^{\alpha}, 0 ; \gamma\right)=I(0,0 ; \gamma)+\left.\frac{\partial I}{\partial u}\right|_{u=0} u+O\left(u^{2}\right) .
$$

The derivative $\partial I / \partial u$ is explicitly given as

$$
\begin{gathered}
\quad \frac{1}{2 \pi} \frac{\partial I}{\partial u}=\alpha \gamma u^{\alpha-1}\left(1+u^{\alpha}\right)^{\gamma-1} \int_{0}^{\infty} d t \int_{1}^{\infty} d s\left[\frac{1}{\left(s+u^{\alpha}\right)^{\gamma}}+\frac{1}{\left(t+u^{\alpha}\right)^{\gamma}}\right] K\left(s, t, u^{\alpha} ; \gamma\right) \\
-\alpha \gamma u^{\alpha-1}\left(1+u^{\alpha}\right)^{\gamma} \int_{0}^{\infty} d t \int_{1}^{\infty} d s\left[\frac{1}{\left(s+u^{\alpha}\right)^{\gamma+1}}+\frac{1}{\left(t+u^{\alpha}\right)^{\gamma+1}}\right] K\left(s, t, u^{\alpha} ; \gamma\right) \\
-\alpha \gamma u^{\alpha-1}\left(1+u^{\alpha}\right)^{\gamma} \int_{0}^{\infty} d t \int_{1}^{\infty} d s\left[\frac{1}{\left(s+u^{\alpha}\right)^{\gamma}}+\frac{1}{\left(t+u^{\alpha}\right)^{\gamma}}\right] K\left(s, t, u^{\alpha} ; \gamma+1\right) .
\end{gathered}
$$

In the limit of $u \rightarrow 0$, only the second term gives a non-vanishing contribution:

$$
\left.\frac{1}{2 \pi} \frac{\partial I}{\partial u}\right|_{u=0}=-\lim _{u \rightarrow 0} \alpha \gamma u^{\alpha-1} \int_{0}^{\infty} d t \int_{1}^{\infty} d s \frac{1}{\left(t+u^{\alpha}\right)^{\gamma+1}}\left[\frac{2 \gamma}{3\left(s+u^{\alpha}\right)^{\gamma+1}} t+O\left(t^{2}\right)\right] .
$$

By setting $t \equiv u^{\alpha} \tau$ and then considering the limit $u \rightarrow 0$, one finds that only the choice of $\alpha=1 /(2-\gamma)$ gives the finite value for equation (14) which is contributed only by the term proportional to $t$ in the square bracket:

$$
\left.\frac{1}{2 \pi} \frac{\partial I}{\partial u}\right|_{u=0}=-\alpha \gamma \int_{1}^{\infty} \frac{2 \gamma d s}{3 s^{\gamma+1}} \int_{0}^{\infty} \frac{\tau d \tau}{(\tau+1)^{\gamma+1}}=-\frac{2}{3(\gamma-1)(2-\gamma)} \quad(1<\gamma<2)
$$


The above method can be also applied to the perturbative analysis for $I(0, w ; \gamma)$ and $I(y, w=y ; \gamma)$, and a straightforward calculation yields

$$
\begin{aligned}
I(y, 0 ; \gamma) & =I(0,0 ; \gamma)-\frac{4 \pi}{3(\gamma-1)(2-\gamma)} y^{2-\gamma}+O\left(y^{4-2 \gamma}\right) \\
I(0, w ; \gamma) & =I(0,0 ; \gamma)-\frac{2 \pi}{2-\gamma} B\left(\frac{5-\gamma}{2}, \frac{\gamma}{2}\right) w^{2-\gamma}+O\left(w^{4-2 \gamma}\right) \\
I(y, y ; \gamma) & =I(0,0 ; \gamma)-\frac{4 \pi y^{2-\gamma}}{3(2-\gamma)} \int_{0}^{\infty} \frac{\tau^{4}\left(\gamma \tau^{2}+3 \tau+\gamma+3\right)}{\left(\tau^{2}+1\right)^{5 / 2}(\tau+1)^{\gamma+1}} d \tau+O\left(y^{4-2 \gamma}\right)
\end{aligned}
$$

where $B(\alpha, \beta)$ is the beta function. The above expressions clearly show the importance of the finite size effect for the observational value $\gamma=1.8$. Since the lowest-order correction term begins with $y^{2-\gamma}$, even a very small value of $y$ changes the value of $I(y, w ; \gamma)$ significantly, which will be discussed in details below.

\section{2. numerical evaluation}

Figure 11 plots the results of the numerical evaluation of equation (10) for $\gamma=1.8$ in comparison with the perturbative expansions (16) to (18). In practice, here and in what follows we set $w=10^{-10}$ and $y=10^{-10}$, respectively, when we numerically compute the values of $I(y, 0 ; \gamma)$ and $I(0, w ; \gamma)$. The perturbation result is in excellent agreement with the numerical evaluation up to $y=0.001$ for $I(y, 0 ; 1.8)$ and $I(y, y ; 1.8)$, and up to $w=0.1$ for $I(0, w ; 1.8)$. Also the perturbation correction turns out to provide a reasonably good approximation even up to $y \sim w \sim 1$. Thus for astrophysically relevant values of $r_{s}$ and $r_{c}$, the perturbation formulae (eqs. 16 to [18]) are very useful if $1<\gamma<2$. It should be noted that even very small values of $y$ and/or $w$ significantly decrease $I(y, w, \gamma)$ relative to the idealistic point-mass approximation $I(0,0, \gamma)=I_{0}(\gamma)$; for $\gamma=1.8, I(y, w, \gamma) / I(0,0, \gamma)$ is about 0.8 even if $y$ or $w$ is $\sim 10^{-3}$. On the other hand, the dependence on $y$ and $w$ is fairly weak as indicated by the perturbation correction (16) to (18). Therefore in a sense

most of the effect might be interpreted as the inherent offset originated from the point-mass approximation itself.

If $\gamma>2$, on the other hand, the above perturbative approach does not work and one should rely on the fully numerical integration. Figures 2 and 3 show such results; $I(y, 0 ; \gamma)$ 
and $I(y, y ; \gamma)$ for various values of $\gamma$. We find the following accurate fitting formulae for $2<\gamma<3$ :

$$
\begin{aligned}
& I(y, 0 ; \gamma)=A\left[\left(y / y_{a}\right)^{\alpha_{a}}+\left(y / y_{a}\right)^{\beta_{a}}\right]^{-1}, \\
& I(y, y ; \gamma)=B\left[\left(y / y_{b}\right)^{\alpha_{b}}+\left(y / y_{b}\right)^{\beta_{b}}\right]^{-1},
\end{aligned}
$$

where

$$
\begin{aligned}
A & =24.99-36.20(\gamma-2)+13.76(\gamma-2)^{2}, \\
y_{a} & =0.197+0.198(\gamma-2)+0.770(\gamma-2)^{2}, \\
\alpha_{a} & =0.0858+0.605(\gamma-2)+0.274(\gamma-2)^{2}, \\
\beta_{a} & =0.747+0.860(\gamma-2)+0.625(\gamma-2)^{2}, \\
B & =19.23-21.65(\gamma-2)+5.807(\gamma-2)^{2}, \\
y_{b} & =0.214+0.134(\gamma-2)+0.278(\gamma-2)^{2}, \\
\alpha_{b} & =0.116+0.561(\gamma-2)+0.260(\gamma-2)^{2}, \\
\beta_{b} & =0.946+1.003(\gamma-2)+0.469(\gamma-2)^{2} .
\end{aligned}
$$

At least in the range of $0.001 \lesssim y \lesssim 1$, the agreement is excellent (Figs. 7 and 3 ) between the numerical results and the fitting formulae.

\section{PREDICTIONS FOR THE SMALL-SCALE VELOCITY DISPERSIONS}

\section{1. power-law models}

Let us consider the predictions for the small-scale velocity dispersions on the basis of the CVT with the finite size effect. With nonzero $r_{s}$ and $r_{c}$, equation (7) should be explicitly rewritten as

$$
\begin{aligned}
\left\langle v_{12}^{2}(r)\right\rangle_{C V T}^{1 / 2} & =1460 \sqrt{\frac{\Omega_{0} Q_{\rho}}{1.3 b_{\mathrm{g}}^{2}}} 5.4^{\frac{\gamma-1.8}{2}}\left(\frac{r_{0}}{5.4 h^{-1} \mathrm{Mpc}}\right)^{\frac{\gamma}{2}} \\
& \times \sqrt{\frac{I\left(r_{c} / r, r_{s} / r ; \gamma\right)}{33.2}}\left(\frac{r}{1 h^{-1} \mathrm{Mpc}}\right)^{\frac{2-\gamma}{2}} \mathrm{~km} / \mathrm{sec} .
\end{aligned}
$$


Due to the $r$-dependence of $I\left(r_{c} / r, r_{s} / r ; \gamma\right),\left\langle v_{12}^{2}(r)\right\rangle_{C V T}^{1 / 2}$ is not described by a power-law in general, but is still proportional to $\sqrt{\Omega_{0} Q_{\rho} r_{0}^{\gamma} / b_{\mathrm{g}}^{2}}$.

Figure 4 plots $\left\langle v_{12}^{2}(r)\right\rangle_{C V T}^{1 / 2} / \sqrt{\Omega_{0} Q_{\rho} / b_{\mathrm{g}}^{2}} /\left(r_{0} / 5.4 h^{-1} \mathrm{Mpc}\right)^{\gamma / 2}$ for $\gamma=1.8$. As discussed in $\S 3$, even fairly small values for $r_{s}$ and $r_{c}$ can reduce the predictions appreciably relative to the case of the point-mass approximation $\left(r_{s}=r_{c}=0\right)$. Furthermore, when $r_{c}>0$, the softening of the force has negligible effect if $r_{s} \leq r_{c}$. Since the observed two-point correlation functions of galaxies do not show any noticeable departure from the single power-law at least for $r \gtrsim 0.02 h^{-1} \mathrm{Mpc}$, the choice of $r_{s}=r_{c}=100 h^{-1} \mathrm{kpc}$ plotted here might be an extreme example, and already overestimate the finite size effect.

Similarly the results for various $\gamma$ are plotted in Figure for $r_{s}=r_{c}=100 h^{-1} \mathrm{kpc}$ (dashed curves) and $r_{s}=r_{c}=10 h^{-1} \mathrm{kpc}$ (solid curves). The results for larger $\gamma$ become more sensitive to the values of $r_{s}$ and $r_{c}$. Given the fact that the point-mass approximation fails for $\gamma>2$, this is reasonable. It should be also pointed out here that even when $\gamma>2$ $\left\langle v_{12}^{2}(r)\right\rangle_{C V T}^{1 / 2}$ increases as $r$ because $I\left(r_{c} / r, r_{s} / r ; \gamma\right)$ increases more strongly than $\propto r^{\gamma-2}$; in the case of $r_{s}=r_{c}=100 h^{-1} \mathrm{kpc}$ and $r \lesssim 1 h^{-1} \mathrm{Mpc}$ relevant for Figure $5,\left\langle v_{12}^{2}(r)\right\rangle_{C V T}^{1 / 2}$ is proportional to $r^{1-\gamma / 2+\beta_{b} / 2}=r^{0.48}, r^{0.51}, r^{0.56}$, and $r^{0.62}$ for $\gamma=2.2,2.4,2.6$, and 2.8 , respectively. When $r / r_{s}, r / r_{c} \gtrsim 10,\left\langle v_{12}^{2}(r)\right\rangle_{C V T}^{1 / 2}$ approaches $\propto r^{1-\gamma / 2+\alpha_{b} / 2}$ and thus significantly levels off (around $r \sim 1 h^{-1} \mathrm{Mpc}$ for $r_{s}=r_{c}=100 h^{-1} \mathrm{kpc}$ and $r \sim 0.1 h^{-1} \mathrm{Mpc}$ for $r_{s}=r_{c}=10 h^{-1} \mathrm{kpc}$ in Fig.(7). In any case, this implies that the scale-dependence of $\left\langle v_{12}^{2}(r)\right\rangle_{C V T}^{1 / 2}$ is fairly insensitive to the value of $\gamma$.

\subsection{COBE normalized CDM models}

The results presented above are fairly general in the sense that they should be valid as long as the CVT is correct and equations (4), (8) and (9) are reasonably good approximation to the reality. Nevertheless the velocity dispersion $\left\langle v_{12}^{2}(r)\right\rangle_{C V T}^{1 / 2}$ is still crucially dependent on the parameters including $\Omega_{0}, Q_{\rho}, b_{\mathrm{g}}, r_{0}, \gamma$ as well as $r_{s}$ and $r_{c}$ which we have discussed in details here. Therefore to proceed further one has to assume some sort of specific models. For this purpose cold dark matter (CDM) models would provide good working hypotheses. 
In particular very accurate semi-analytical fitting formulae for $\xi_{\rho}(r)$ have been worked out in the literature, which have been successfully tested against high-resolution N-body simulations (Hamilton et al. 1991; Jain, Mo \& White 1995; Peacock \& Dodds 1994, 1996; Mo, Jing \& Börner 1996).

CDM models with the primordial scale-invariant (Harrison -Zel'dovich) spectrum have the power spectrum $P(k)$ which is asymptotically proportional to $k^{-3}$ for large $k$. This implies that the two-point correlation function in these models should approach constant on the corresponding scales; for a purely scale-free primordial spectra of $P(k) \propto k^{n}, \xi_{\rho}(r)$ is proportional to $r^{-3-n}$ in a linear regime and to $r^{-3(n+3) /(n+5)}$ in a stable clustering (strongly nonlinear) regime. In other words, we naturally expect the small-scale flattening of the form (9) even independently of the finite size effect of galaxies. This is another reason why the present analysis is very important in realistic cosmological scenarios.

Given the cosmological parameters $\Omega_{0}, \lambda_{0}$, and $h$, the normalization constant of the fluctuation spectrum can be determined by COBE data (e.g., Sugiyama 1995), and thus the (non-linear) mass correlation function $\xi_{\rho}(r)$ can be analytically calculated (Peacock \& Dodds 1996; Mo et al. 1996). We then determine $r_{0}, \gamma$ and $r_{c}$ by fitting the calculated $\xi_{\rho}(r)$ to equation (9). So determined $r_{c}$ does not represent the finite size effect of galaxies, and if it is below $10 h^{-1} \mathrm{kpc}$, for instance, we may set $r_{s}=r_{c}=10 h^{-1} \mathrm{kpc}$ to include the effect. Therefore as long as the hierarchical relation (4) holds for the two- and three-point correlation functions of mass, the only remaining parameter is $Q_{\rho}$. Although recent numerical and semi-analytical studies indicate that $Q_{\rho}$ is weakly dependent on the scale (Suto 1993; Matsubara \& Suto 1994; Suto \& Matsubara 1994; Jing \& Börner 1996; Mo et al. 1996), it is about $2 \sim 3$ on scales less than $1 h^{-1} \mathrm{Mpc}$.

We use the following expression for the COBE $2 \mathrm{yr}$ data normalization $\sigma_{\mathrm{COBE}}\left(8 h^{-1} \mathrm{Mpc}\right)$ by Nakamura (1996) who performed a fit to the numerical results by Sugiyama (1995):

$$
\begin{aligned}
& \sigma_{\mathrm{COBE}}\left(8 h^{-1} \mathrm{Mpc}\right)=(30 \Gamma)^{2} f(8 \Gamma) \\
& \times \begin{cases}\sqrt{1+1.22\left(1-\Omega_{0}\right)-0.266\left(1-\Omega_{0}\right)^{2}-1.23\left(1-\Omega_{0}\right)^{3}} & \left(\lambda_{0}=0\right) \\
\Omega_{0}^{-0.696} & \left(\lambda_{0}=1-\Omega_{0}\right)\end{cases}
\end{aligned}
$$


where

$$
\begin{aligned}
\Gamma & \equiv \Omega_{0} h \exp \left[-\Omega_{b}\left(1+\frac{\sqrt{2 h}}{\Omega_{0}}\right)\right] \\
f(x) & \equiv\left(18.1 x^{0.2}+7.17 x^{0.5}+22.1 x+1.23 x^{2}\right)^{-1}
\end{aligned}
$$

For definiteness we adopt the baryon density parameter $\Omega_{b}=0.015 h^{-2}$. Note that the COBE 2yr data give a slightly larger normalization amplitude (roughly by 10 percent) compared with the COBE 4yr data (Sugiyama 1996, in private communication). Our conclusions below, however, are insensitive to this issue.

Figure 6 plots the two-point correlation functions of mass based on the Peacock \& Dodds (1996) model and our fits to the form (9) for the CDM models in Table 1. The parameter fitting is performed over the range $0.01 h^{-1} \mathrm{Mpc} \lesssim r \lesssim 5 h^{-1} \mathrm{Mpc}$. Although the fit does not seem so good especially on small scales, any single power-law form (i.e., $r_{c}=0$ ) yields much poorer fits and the inclusion of the nonzero $r_{c}$ definitely improve the theoretical prediction.

Figure 7 shows $\left\langle v_{12}^{2}(r)\right\rangle_{C V T}^{1 / 2} / \sqrt{Q_{\rho}}$ for the three CDM models in Table 1 . The model parameters except for $Q_{\rho}$ are completely determined from theory and the fitting, and therefore the result is fairly definite. As mentioned above, recent numerical and semi-analytical results suggest $Q_{\rho}=(2 \sim 3)$ on scales less than $1 h^{-1} \mathrm{Mpc}$. With this value in mind, Figure 7 implies that $\Omega_{0}=1 \mathrm{CDM}$ models disagree with the observed values $(300 \sim 600) \mathrm{km} / \mathrm{sec}$ at $r=1 h^{-1} \mathrm{Mpc}$ (Davis \& Peebles 1983; Mo, Jing \& Börner 1993; Fisher et al. 1994; Marzke et al. 1995$)$ by a factor of $(3 \sim 6)$. Although the conflict with the

Table 1: Fitted parameters for CDM models

\begin{tabular}{rrrcccc}
\hline$\Omega_{0}$ & $\lambda_{0}$ & $h$ & $\sigma_{\mathrm{COBE}}\left(8 h^{-1} \mathrm{Mpc}\right)$ & $\gamma$ & $r_{0}\left(h^{-1} \mathrm{Mpc}\right)$ & $r_{c}\left(h^{-1} \mathrm{Mpc}\right)$ \\
\hline \hline 1 & 0 & 0.5 & 1.35 & 2.002 & 7.67 & 0.061 \\
0.2 & 0 & 0.7 & 0.27 & 1.42 & 0.79 & 0.0 \\
0.2 & 0.8 & 0.7 & 0.78 & 1.99 & 4.67 & 0.087 \\
\hline
\end{tabular}


$\Omega_{0}=1$ model has been pointed out frequently in the literature (e.g., Peebles 1976, 1980; Davis \& Peebles 1983; Suto 1993), our present prediction is the most precise one from a theoretical point of view. Finally in Figure 8, we plot $\left\langle v_{12}^{2}\left(r=0.8 h^{-1} \mathrm{Mpc}\right)\right\rangle_{C V T}^{1 / 2} / \sqrt{Q_{\rho}}$ as a function of $\Omega_{0}$ both for $\lambda_{0}=0$ and $1-\Omega_{0}$ models using the same procedure described here. Clearly only the low-density models are compatible with the observations even if one allows for a factor of $(2 \sim 3)$ uncertainty of the sample-to-sample variation (Suto 1996; Suto \& Jing 1996). It is interesting to note from the figure that softening in the force with

$r_{s} \approx 100 h^{-1} \mathrm{kpc}$ has almost negligible effect on the prediction of $\left\langle v_{12}^{2}\right\rangle^{1 / 2}$ in the three CDM models; this is simply because these models have either $\gamma \ll 2$ or $r_{c} \sim 100 h^{-1} \mathrm{kpc}$.

\section{DISCUSSION AND CONCLUSIONS}

We have presented a realistic refinement of the cosmic virial theorem by taking into account of the finite size effect of galaxies. The CVT in its conventional form which treats galaxies as point particles appreciably overestimates small-scale velocity dispersions of galaxies. Although this is already remarked by Peebles (1976) and in a slightly different context by Bartlett \& Blanchard (1996), we presented more detailed analysis on the basis of a perturbation theory and systematic numerical calculations. In particular the former provides a clear physical insight into the finite size effect. Our perturbation formulae (eqs.16-18), which are accurate enough [better than $20 \%$ in accuracy for $I(y, w ; \gamma)$ ] for most purposes, can be easily and reliably used to correct for the flattening of mass correlation $\xi_{\rho}(r)$ and the force softening for $\gamma<2$. We have also presented accurate fitting formulae for $I(y, w ; \gamma)$ for the case of $\gamma>2$. Moreover, we have predicted the pairwise velocity dispersion for CDM models with the COBE fluctuation normalization.

As our result indicates that the flattening of mass correlation $\xi_{\rho}(r)$ has significant effect on the CVT, one should be very cautious to infer $\Omega_{0}$ from observational measurements of galaxy-galaxy correlation function $\xi_{g}(r)$ and galaxy pairwise velocity dispersions. Although current observations of galaxy clustering have not revealed any noticeable flattening in $\xi_{g}(r)$ on small scales, non-linear biasing mechanisms may have complicated the relation 
between $\xi_{g}(r)$ and $\xi_{\rho}(r)$. Moreover, the statistical significance of galaxy clustering on very small scales $r \lesssim 200 h^{-1} \mathrm{kpc}$ is still rather poor. In fact, intrinsic flattening of correlation functions on small scales is expected generally for CDM models with a Harrison -Zel'dovich primordial spectrum. We have quantitatively pointed out the importance of taking into account such flattening in $\xi_{\rho}(r)$ when one applies the CVT, otherwise the estimated value for $\Omega_{0}$ would be substantially underestimated.

To avoid the complicated issue of possible non-linear biasing on small scales, one can draw constraints on $\Omega_{0}$ from a comparison of the measured pairwise velocity dispersion with our predictions for CDM models of various $\Omega_{0}$ (See also Mo et al. 1996). As long as one relies on the COBE normalization, our CDM predictions are fairly definite; for realistic $\mathrm{CDM} \xi_{\rho}(r)$ with flattening, fortunately, the (unknown) force softening even with $r_{s} \lesssim 100 h^{-1} \mathrm{kpc}$ would not make any difference on the prediction of $\left\langle v_{12}^{2}\right\rangle^{1 / 2}$ (see $\S 4.2$ and Fig.(7). The remaining two parameters which would complicate the comparison between the predictions and observations include the normalized amplitude of the three-point correlation functions which is unlikely to be determined accurately from a small sample of galaxies, and a possible velocity bias of galaxies which may result from the dynamical friction but whose quantitative effect is still controversial in literature.

Although the present analysis provides a theoretical improvement over the conventional CVT, the usefulness of the CVT to estimate $\Omega_{0}$ also relies on the quality of the observational data. In fact, Mo et al. (1993) noted that the observed velocity dispersions vary fairly significantly from sample to sample, and also are sensitive to the modeling of the data. We will discuss this topic in details elsewhere (Suto \& Jing 1996).

We thank Houjun Mo and Gerhard Börner for useful discussions and comments on the manuscript, and Takahiro T. Nakamura for providing the fitting formula for the COBE 2yr normalization. YPJ gratefully acknowledges the receipt of an Alexander-von-Humboldt research fellowship. YS is grateful to Gerhard Börner for hospitality at Max-Planck-Institut für Astrophysik, where the present work was done. This research was supported in part by the Grants-in-Aid by the Ministry of Education, Science, Sports and Culture of Japan (07CE2002) to RESCEU (Research Center for the Early Universe). 


\section{REFERENCES}

Ballinger, W.E., Peacock, J.A. \& Heavens, A.F. 1996, MNRAS, in press.

Bartlett, J. G. \& Blanchard, A. 1996, A\&A, 307, 1

Davis, M. \& Peebles, P.J.E. 1983, ApJ, 267, 465

Fisher, K.B., Davis,M., Strauss, M.A., Yahil, A., \& Huchra, J. 1994, MNRAS, 267, 927

Hamilton, A.J.S., Kumar, P., Lu, E., \& Matthews, A. 1991, ApJ, 374, L1

Hamilton, A.J.S. 1992, ApJ, 385, L5

Jain, B., Mo, H.J., \& White, S.D.M. 1995, MNRAS, 276, L25

Jing, Y.P. \& Börner, G. 1996, A\&A, in press.

Kaiser, N. 1987, MNRAS, 227, 1

Marzke,R.O., Geller, M.J., da Costa, L.N., \& Huchra,J. 1995, AJ, 110, 477

Matsubara, T. \& Suto, Y. 1994, ApJ, 420, 497

Matsubara, T. \& Suto, Y. 1996, ApJ October 10 issue, in press.

Mo, H.J., Jing, Y.P., \& Börner, G. 1993, MNRAS, 264, 825

Mo, H.J., Jing, Y.P., \& Börner, G. 1996, MNRAS, submitted.

Nakamura, T.T. 1996, master thesis to the University of Tokyo, unpublished.

Peacock, J.A. \& Dodds, S.J. 1994, MNRAS, 267, 1020

Peacock, J.A. \& Dodds, S.J. 1996, MNRAS, 280, L19

Peebles, P.J.E. 1976, Astrophys.Sp.Sci., 45, 3

Peebles, P.J.E. 1980, The Large Scale Structure of the Universe (Princeton University Press: Princeton)

Sugiyama,N., 1995, ApJS, 100, 281

Suto, Y. 1993, Prog.Theor.Phys., 90, 1173

Suto, Y. 1996, in Proceedings of the inauguration conference of Asia Pacific Center for Theoretical Physics eds. J.W.Kim, (World Scientific: Singapore), in press

Suto, Y. \& Jing, Y.P. 1996, in preparation.

Suto, Y. \& Matsubara, T. 1994, ApJ, 420, 504

Suto, Y. \& Matsubara, T. 1996, ApJ, submitted. 


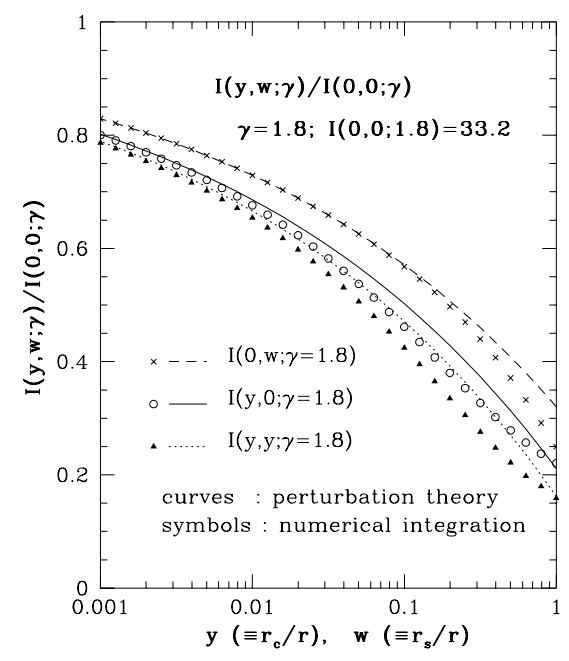

Fig. 1. - Effect of nonzero $y$ and $w$ on $I(y, w ; \gamma)$ for $\gamma=1.8$. Symbols indicate the results of direct numerical evaluations while thin curves plot those of the perturbation theory.

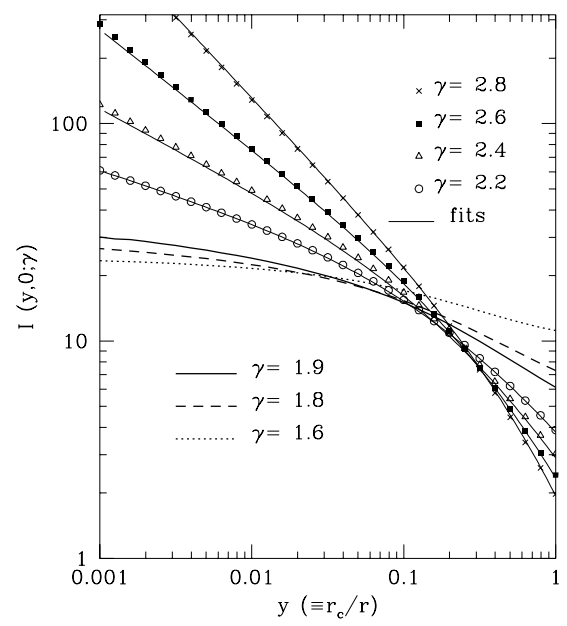

Fig. 2.- $I(y, 0 ; \gamma)$ for different $\gamma\left(r_{s}=0\right)$. Thick curves indicate for $\gamma<2$ while symbols (direct numerical integration) and thin solid curves (fitting formula) for $\gamma>2$. 


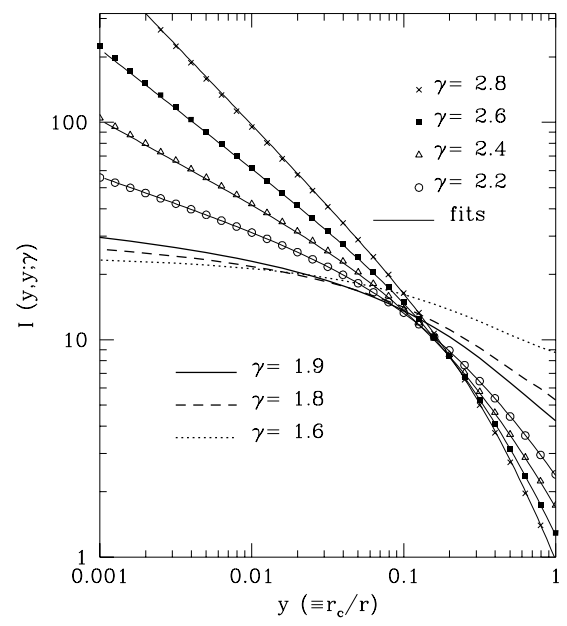

Fig. 3.- $I(y, y ; \gamma)$ for different $\gamma\left(r_{s}=r_{c}\right)$. Thick curves indicate $\gamma<2$ while symbols (direct numerical integration) and thin solid curves (fitting formula) for $\gamma>2$.

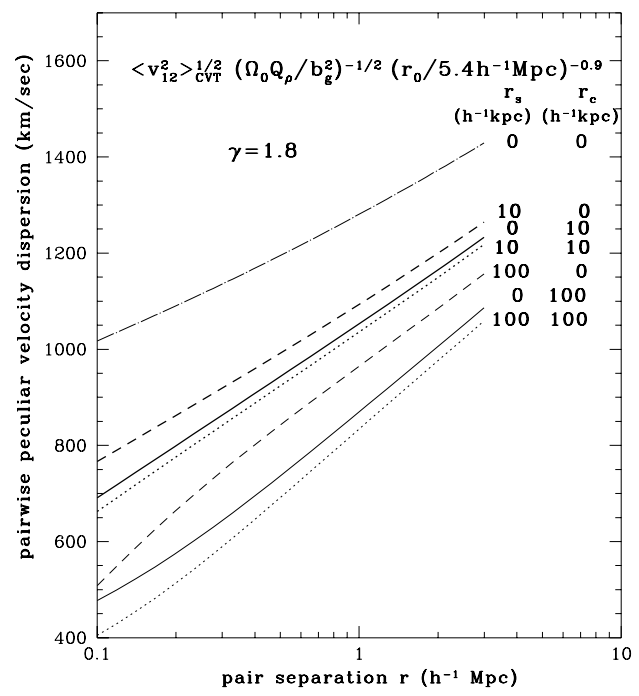

Fig. 4.- Relative pairwise peculiar velocity dispersions predicted from the CVT $(\gamma=1.8)$. Different curves correspond to different sets of values for $r_{s}$ and $r_{c}$ indicated in the panel. 


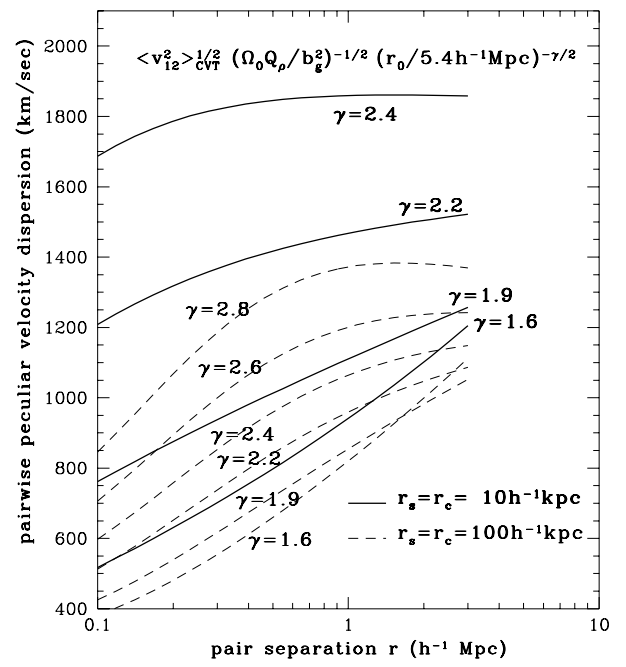

Fig. 5.- Relative pairwise peculiar velocity dispersions predicted from the CVT for various $\gamma$. Solid and dashed curves correspond to $r_{s}=r_{c}=10 h^{-1} \mathrm{kpc}$ and $100 h^{-1} \mathrm{kpc}$, respectively.

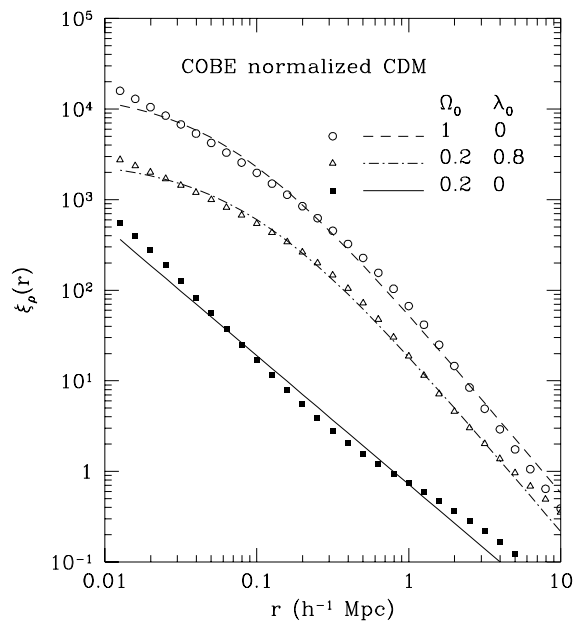

Fig. 6.- Two-point correlation functions of mass for the three CDM models in Table 1 computed from analytical models (symbols) and the power-law fits with nonzero $r_{c}$ (curves); $\Omega_{0}=1.0$ and $\lambda_{0}=0.0$ (open circles and dashed curve), $\Omega_{0}=0.2$ and $\lambda_{0}=0.0$ (filled squares and solid curve), and $\Omega_{0}=0.2$ and $\lambda_{0}=0.8$ (open triangles and dot-dashed curve). 


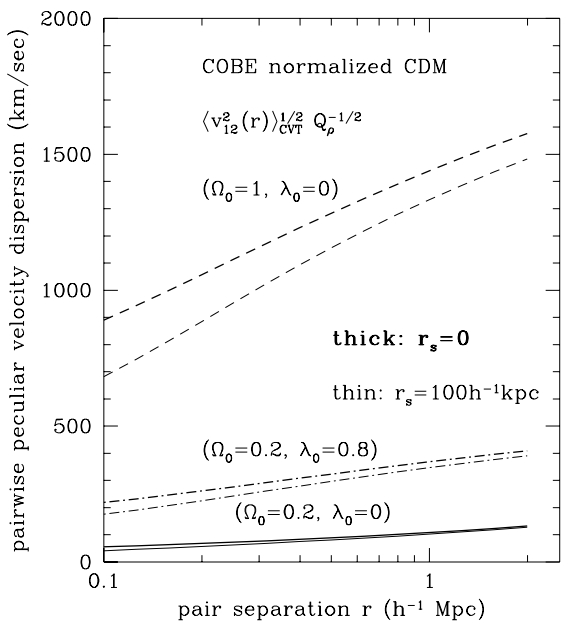

Fig. 7.- Relative pairwise peculiar velocity dispersions from the CVT as functions of $r$ for the three CDM models in Table 1. $\Omega_{0}=1.0$ and $\lambda_{0}=0.0$ (dashed curve), $\Omega_{0}=0.2$ and $\lambda_{0}=0.8$ (dot-dashed curve), and $\Omega_{0}=0.2$ and $\lambda_{0}=0.0$ (solid curve). Thick curves indicate for $r_{s}=0$, while thin curves for $r_{s}=100 h^{-1} \mathrm{kpc}$.

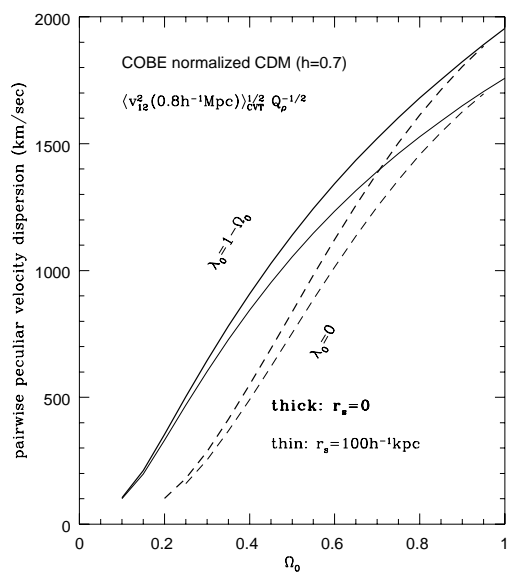

Fig. 8.- Relative pairwise peculiar velocity dispersions at $r=0.8 h^{-1} \mathrm{kpc}$ as functions of $\Omega_{0}$ for COBE normalized CDM models $(h=0.7) ; \lambda_{0}=0$ models in dashed and $\lambda_{0}=1-\Omega_{0}$ models in solid curves. Thick curves indicate for $r_{s}=0$, while thin curves for $r_{s}=100 \mathrm{~h}^{-1} \mathrm{kpc}$. 